\title{
Pathological characteristics of BK polyomavirus-associated nephropathy with glomerular involvement
}

\author{
Xu-Tao Chen ${ }^{1,2,3 \#}$, Rong-Hai Deng ${ }^{1,2,3 \#}$, Shi-Cong Yang ${ }^{4 \#}$, Xiao-Tao Hou ${ }^{5}$, Jun Li ${ }^{1,2,3}$, Yan-Yang Chen ${ }^{4}$, \\ Hui-Fei Yang $^{6}$, Ze-Yuan Wang ${ }^{7}$, Chang-Xi Wang ${ }^{1,2,3}$, Jiang Qiu ${ }^{1,2,3}$, Li-Zhong Chen ${ }^{1,2,3}$, Wen-Fang Chen ${ }^{4 *}$, \\ Gang Huang ${ }^{1,2,3 *}$
}

${ }^{1}$ Department of Organ Transplantation, The First Affiliated Hospital, ${ }^{2}$ Guangdong Provincial Key Laboratory of Organ Donation and Transplant Immunology, The First Affiliated Hospital, ${ }^{3}$ Guangdong Provincial International Cooperation Base of Science and Technology (Organ Transplantation), The First Affiliated Hospital, ${ }^{4}$ Department of Pathology, The First Affiliated Hospital, Sun Yat-sen University, Guangzhou, China; ${ }^{5}$ Guangzhou KingMed Center for Clinical Laboratory Co., Ltd., Guangzhou, China; ${ }^{6}$ Fuda Cancer Hospital, Jinan University, Guangzhou, China; ${ }^{7}$ Zhongshan School of Medicine, Sun Yat-Sen University, Guangzhou, China

Contributions: (I) Conception and design: XT Chen, WF Chen, G Huang; (II) Administrative support: LZ Chen, CX Wang; (III) Provision of study materials or patients: G Huang, XT Chen, RH Deng, J Li, LZ Chen, CX Wang; (IV) Collection and assembly of data: SC Yang, XT Hou, YY Chen, HF Yang, XT Chen; (V) Data analysis and interpretation: XT Chen, RH Deng, ZY Wang; (VI) Manuscript writing: All authors; (VII) Final approval of manuscript: All authors.

\#These authors contributed equally to this work as co-first authors.

*These authors contributed equally to this work as co-corresponding authors.

Correspondence to: Gang Huang, PhD; Wen-Fang Chen, PhD. 58\# Zhongshan Road 2, Guangzhou 510080, China.

Email: huanggang_791021@163.com; chwfang@mail.sysu.edu.cn.

Background: This study aimed to investigate the pathological characteristics of BK polyomavirus $(\mathrm{BKPyV})$-associated nephropathy (BKPyVAN) with glomerular involvement in kidney transplant recipients.

Methods: Forty-four patients with glomerular $\mathrm{BKPyV}$ infection were retrospectively included for analysis. Immunohistochemical (IHC) staining was performed on paraffin sections using monoclonal mouse antiSV40 large T antigen antibody.

Results: In BKPyV-infected glomeruli, the glomerular parietal epithelial cells (GPECs) were swollen, hyperchromatic, and enlarged, with an increased nuclear to cytoplasm (N/C) ratio and smudgy basophilic intra-nuclear viral inclusions. IHC staining revealed the distribution of BKPyV involvement in GPECs, podocytes, and shedding cells within Bowman's space. Notably, BKPyV affected GPEC proliferation and caused crescent formation (7 biopsies, 15.9\%). Three biopsies exhibited fibrous crescents and the absence of viral inclusions. The other 4 biopsies exhibited cellular and fibro-cellular crescents, with viral cytopathic changes and positive IHC staining in the proliferative GPECs. Electron microscopy showed viral particles in both GPECs and podocytes. BKPyV-infected GPECs were degenerative, with mitochondrial swelling, endoplasmic reticulum expansion, and multi-layered membranous structure formation. Twelve (27.3\%) patients received repeat biopsies within 1.6 to 39.5 months (median: 13.5 months), but none revealed persistent glomerular $\mathrm{BKPyV}$ infection.

Conclusions: Distinct glomerular changes in BKPyVAN biopsies should raise the possibility of glomerular involvement.

Keywords: BK polyomavirus (BKPyV); kidney transplantation; glomerular infection; pathological characteristics

Submitted Feb 24, 2020. Accepted for publication Jul 10, 2020.

doi: 10.21037/atm-20-1879

View this article at: http://dx.doi.org/10.21037/atm-20-1879 


\section{Introduction}

BK polyomavirus (BKPyV)-associated nephropathy (BKPyVAN) is a known cause of renal allograft dysfunction and failure (1). Renal biopsy is considered the gold-standard diagnostic test, showing distinct intra-nuclear viral inclusions and SV40 large $T$ antigen staining (2). In most biopsies, $\mathrm{BKPyV}$ is detected within the tubular epithelium, and occasionally in Bowman's capsular parietal epithelium. $\mathrm{BKPyV}$ infection often begins in the collecting tubules and distal convoluted tubules in the medulla, and gradually spreads to the tubules in the cortical area. In mild or moderate cases, $\mathrm{BKPyV}$ infection is confined to the tubules $(3,4)$. In advanced stages, $\mathrm{BKPyV}$ can infect the glomeruli; this is infrequent but can cause irreversible injury to the kidney. The presence of viral cytopathic changes in the glomeruli has also been associated with a poor prognosis (5). Similarly, we have found $\mathrm{BKPyV}$ infection in glomerular parietal epithelial cells (GPECs) in some kidney transplant (KT) recipients, and these patients had rapid deterioration of renal function and even graft loss (6). BKPyVAN with glomerular involvement exhibits specific pathological features. The glomerular changes caused by BKPyV infection are particularly distinctive but have not been described in detail.

Thus, the purpose of this study was to describe in detail the pathological characteristics of BKPyVAN with glomerular involvement. We present the following article in accordance with the STROBE reporting checklist (available at http://dx.doi.org/10.21037/atm-20-1879).

\section{Methods}

\section{Clinical data}

With the approval from the local Institutional Review Board, we retrospectively reviewed the renal transplant biopsies performed at our center from 1/1/2008 to 9/30/2018. During this period, 189 renal transplant recipients, who received 288 biopsies, were diagnosed with BKPyVAN and GPEC involvement was observed in 44 patients. The pathological findings of patients with glomerular involvement were retrospectively analyzed. The study was conducted in accordance with the Declaration of Helsinki (as revised in 2013). The study was approved by the Ethics Committee and the Research Board of our institution [approval No. (2009) 28]. Because of the retrospective nature, the requirement of informed consent was waived.

\section{Pathological methods}

Hematoxylin-eosin, periodic acid Schiff, Masson's trichrome, and methenamine silver staining were performed for light microscopy evaluation. Immunofluorescence (IF) studies were performed with antibodies against IgA, IgM, IgG, C3, C1q, fibrinogen, and C4d. Nuclear expression of SV40 large $\mathrm{T}$ antigen was confirmed by immunohistochemical (IHC) staining with mouse antiSV40 large $T$ antigen monoclonal antibody (Oncogene Research Products, Cambridge, MA, USA, catalogue number DP02, clone PAb 416) as previously described (7).

\section{Electron microscopy}

A CM10 electron microscope (JEM-1400, Japanese) was used to detect viral particles, especially in tubular epithelial cells and glomerular innate cells. Infected cells were defined as those with viral particles with a diameter between 30 and $45 \mathrm{~nm}$ detected in the cytoplasm or nucleus.

\section{Diagnosis of BKPyVAN}

The diagnosis of polyomavirus-associated nephropathy (PyVAN) was established by the presence of interstitial inflammation, tubular atrophy, interstitial fibrosis, and the existence of viral cytopathic changes in the tubular epithelial cells, confirmed by IHC nuclear positivity with anti-SV40 large $\mathrm{T}$ antigen monoclonal antibody, as previously described (8). The histological features of PyVAN were classified using the American Society of Transplantation (AST) schema, and PyVAN was classified as stage A, B, and $\mathrm{C}$ based on the guidelines published by Hirsch et al. (7). Histological viral load was assessed semi-quantitatively as the percentage of tubules positive for polyomavirus using a 4-tier system (<10\%, 10-25\%, 25-50\%, and >50\%) (9). The diagnosis of BKPyVAN was confirmed on the basis of positive $\mathrm{BK}$ viruria and/or BK viremia, in addition to positive IHC staining for SV40 large T antigen in renal tissue. Particular attention was paid to the presence of anti-SV40 large T antigen staining in Bowman's capsular epithelium. GPEC+ PyVAN was defined as at least 1 glomerular intrinsic cell positive for anti-SV40 large $\mathrm{T}$ antigen. Glomerulitis is characterized by complete or partial occlusion of $\geq 1$ glomerular capillary with leukocyte infiltration and endothelial cell enlargement (10). All slides were reviewed by two independent pathologists, and histological scores were evaluated using the Banff criteria $(10,11)$. 


\section{Statistical analysis}

Continuous data were expressed as mean \pm standard deviation (SD) and compared by Student's $t$-test. If normality was not assumed, continuous data were expressed as median (range), and the Mann-Whitney $U$ test was used for comparisons between groups. Categorical data were indicated by number and percentage (\%) and compared by Pearson's chi-square test or Fisher's exact test between groups. All statistical tests were two-tailed, and a value of $\mathrm{P}<0.05$ was considered to indicate statistical significance. All analyses were performed by using IBM SPSS version 20 software (SPSS Statistics V20, IBM Corporation, Somers, New York, USA).

\section{Results}

\section{Baseline information}

The collected biopsies came from 27 women and 17 men, ranging in age from 21 to 62 years (median: 39.2 years). The native renal diseases included IgA nephropathy $(\mathrm{n}=6)$, hypertension $(\mathrm{n}=1)$, systemic lupus erythematosus (SLE) nephritis $(\mathrm{n}=1)$, and chronic glomerulonephritis with an unknown cause $(n=36)$. The immunosuppressive maintenance regimen included tacrolimus, mycophenolic acid, and prednisone. The reasons for biopsies included serum creatinine elevating $\geq 30 \%$ than baseline value combined with BKPyV infection (40/44, 90.9\%), plasma $\mathrm{BKPyV}$ load $\geq 1,000$ copies/ml (3/44, $6.8 \%)$, and serum creatinine elevating $\geq 30 \%$ than baseline value combined with proteinuria $(1 / 44,2.3 \%)$. At diagnosis of BKPyVAN, the urine $\mathrm{BK}$ viral load varied from $2.7 \times 10^{6}$ to $1.0 \times 10^{11}$ copies $/ \mathrm{mL}$ (median: $1.8 \times 10^{9}$ copies $/ \mathrm{mL}$ ), and plasma BK viral load varied from 0 to $2.1 \times 10^{7}$ copies $/ \mathrm{mL}$ (median: $1.8 \times 10^{4}$ copies $/ \mathrm{mL}$ ). The mean serum creatinine level was $266.6 \pm 138.1 \mu \mathrm{mol} / \mathrm{L}$. Urine test results were presented in Table S1. During a follow-up period of median 14.4 months (interquartile range: $17.6-42.3$ months), 20 (45.5\%) patients lost their graft function.

\section{Light microscopy findings}

The basic pathological features of the 44 patients with glomerular $\mathrm{BKPyV}$ involvement are shown in Table 1. Typical cytopathic evidence of $\mathrm{BKPyV}$ infection, such as intra-nuclear viral inclusions and ground-glass like nuclei were observed in the parietal epithelium of Bowman's capsule, proximal tubular epithelium, and distal tubular epithelium. The glomerular sclerosis ratio ranged from zero to 12.8 . The average number of non-sclerotic glomeruli was $11.0 \pm 10.5$. Mild glomerulitis (Banff g1) was observed in $43.2 \%$ of the biopsies (Figure S1). Mononuclear inflammatory cells were mainly found in the glomerular capillary wall, and occasionally among the proliferating GPECs. Mild mesangial hyperplasia of glomeruli was also observed. Neither endothelial proliferation nor necrosis of glomerular capillary loops was identified. In some cases, glomerular ischemia and shrinkage, capsular thickening, and peri-glomerular fibrosis were observed. No viral inclusions were found in glomerular capillary endothelial cells. All biopsies exhibited mild to severe tubulitis (Banff t1 to t3) in non-atrophic intact tubules, with focal or diffuse interstitial inflammation (Banff i1 to i3; mainly lymphocytes, eosinophils, and plasma cells) (Table 2). Mild to severe interstitial fibrosis and tubular atrophy was also noted in all biopsies (Table 2).

In BKPyV-infected glomeruli, the GPECs were swollen, hyperchromatic, and enlarged, with an increased nuclear to cytoplasm (N/C) ratio, and showed typical $\mathrm{BKPyV}$ infection changes such as ground-glass nuclei and smudgy basophilic intra-nuclear viral inclusions (Figure 1A). The $\mathrm{BKPyV}$-infected GPECs were continuous with the proximal tubular epithelium. Scattered cell debris was observed in the glomerular space. IHC staining for SV40 large T antigen showed mild to moderate positivity in tubular epithelial cell nuclei (Table 1), the parietal epithelium of Bowman's capsule, and the contiguous proximal tubular epithelium (Figure 1B). In two biopsy specimens, viral inclusions were aggregated within the GPECs and the podocytes (Figure 1C), and SV40 large $T$ antigen staining was positive in shedding cells in Bowman's space and podocytes (Figure 1D). There was no positive IHC staining for cytomegalovirus.

Notably, BKPyV could affect GPEC proliferation and cause crescent formation (7 biopsy specimens, 15.9\%). Three biopsies specimens exhibited only fibrous crescents and the absence of viral inclusions. Four specimens had cellular and fibro-cellular crescents, with evident viral cytopathic changes (Figure 2A), and positive SV40 large T staining in the proliferative GPECs (Figure $2 B$ ). In particular, one patient had crescent formation in 11 of 26 glomeruli, including 4 cellular, 2 fibro-cellular, and 5 fibrous crescents. In BKPyV infections, the cellular components exhibited smudgy basophilic inclusions. The involved glomeruli showed no disruption of the capillary basement membranes. 
Table 1 Proportion of various histopathologic lesion in 44 BKPyVAN patients with glomerular involvement

\begin{tabular}{|c|c|}
\hline Pathological features & $\mathrm{N}(\%)$ \\
\hline \multicolumn{2}{|l|}{ Glomerulitis } \\
\hline g0 & $25(56.8)$ \\
\hline$g 1$ & $19(43.2)$ \\
\hline \multicolumn{2}{|c|}{ Chronic transplant glomerulopathy } \\
\hline $\operatorname{cg} 0$ & $42(95.5)$ \\
\hline $\operatorname{cg} 1$ & $1(2.3)$ \\
\hline $\operatorname{cg} 2$ & $1(2.3)$ \\
\hline Crescents & $7(15.9)$ \\
\hline Peri-glomerular fibrosis & $29(65.9)$ \\
\hline \multicolumn{2}{|c|}{ Extent of SV40 large T antigen } \\
\hline $1+$ & $6(13.6)$ \\
\hline $2+$ & $12(27.3)$ \\
\hline $3+$ & $21(47.7)$ \\
\hline $4+$ & $5(11.4)$ \\
\hline \multicolumn{2}{|l|}{ Tubulitis } \\
\hline $\mathrm{t} 1$ & $25(56.8)$ \\
\hline t2 & $13(29.5)$ \\
\hline t3 & $6(13.6)$ \\
\hline \multicolumn{2}{|l|}{ Interstitial inflammation } \\
\hline i0 & $9(20.5)$ \\
\hline ¡1 & $18(40.9)$ \\
\hline i2 & $10(22.7)$ \\
\hline ¡3 & $7(15.9)$ \\
\hline \multicolumn{2}{|l|}{ Tubular atrophy } \\
\hline ct1 & $11(25.0)$ \\
\hline ct2 & $27(61.4)$ \\
\hline ct3 & $6(13.6)$ \\
\hline \multicolumn{2}{|l|}{ Interstitial fibrosis } \\
\hline ci1 & $11(25.0)$ \\
\hline ci2 & $23(52.3)$ \\
\hline ci3 & $10(22.7)$ \\
\hline \multicolumn{2}{|l|}{ Peri-tubular capillaritis } \\
\hline pct0 & $25(56.8)$ \\
\hline ptc1 & $17(38.6)$ \\
\hline
\end{tabular}

Table 1 (continued)
Table 1 (continued)

\begin{tabular}{ll}
\hline Pathological features & $\mathrm{N}(\%)$ \\
\hline ptc2 & $2(4.5)$ \\
Endarteritis & 0 \\
BKPyVAN stage & \\
Stage A & 0 \\
Stage B1 & $8(18.2)$ \\
Stage B2 & $11(25.0)$ \\
Stage B3 & $20(45.5)$ \\
Stage C & $5(11.4)$ \\
CNI nephrotoxicity & $2(4.5)$ \\
FSGS & $2(4.5)$ \\
PDG & $1 / 44(2.3)$ \\
\hline
\end{tabular}

BKPyVAN, BK polyomavirus-associated nephropathy; Ptc, peri-tubular capillaritis; CNI, calcineurin inhibitor; FSGS, focal segmental glomerular sclerosis; PDG, pediatric donor glomerulopathy.

Table 2 The scores of tubular interstitial lesion in 44 patients with glomerular BKPyV involvement

\begin{tabular}{lc}
\hline Pathological indicators & Banff grading scores \\
\hline Extent of SV40 large T antigen & $2.6 \pm 0.9$ \\
Tubulitis & $1.5 \pm 0.8$ \\
Interstitial inflammation & $1.3 \pm 1.0$ \\
Tubular atrophy & $1.9 \pm 0.7$ \\
Interstitial fibrosis & $2.0 \pm 0.7$ \\
\hline
\end{tabular}

BKPyV, BK polyomavirus.

\section{IF staining}

IF staining results are shown in Table 3. Sparse granular deposition of $\operatorname{IgA}$, IgG, IgM, C3, C1q, and fibrinogen was occasionally observed in the mesangium but did not exhibit a particular pattern in glomeruli with or without $\mathrm{BKPyV}$ infection. In 18 (40.9\%) of the biopsy specimens, sparse C4d staining was found along glomerular or tubule basement membranes. No C4d deposition was found along peri-tubular capillaries. The frequency of $\mathrm{C} 4 \mathrm{~d}$ in tubular basement membranes and glomerular basement membranes (GBMs) was similar between viremia (9/14) and nonviremia cases $(15 / 30)(\mathrm{P}=0.375)$. No immunoglobulins or complements were found in Bowman's capsule. 



Figure $1 \mathrm{BK}$ polyomavirus (BKPyV) infection in glomeruli. (A) The BKPyV-infected glomerular parietal epithelial cells presented with typical cytological changes in the peripheral tubular epithelium, with enlarged and ground-glass like nuclei and intra-nuclear inclusions (arrow) [hematoxylin-eosin (HE), x400]. (B) Immunohistochemistry (IHC) staining showed SV40 large T antigen positivity in the BKPyVinfected glomerular parietal epithelial cells, and the contiguous proximal tubular epithelium (B, IHC, $\times 400)$. (C,D) BKPyV infection in both the visceral and parietal epithelium of Bowman's capsule, with enlarged and hyperchromatic nuclei (C, HE, $\times 400)$ and SV40 large T antigen expression (D, IHC, $\times 400)$.
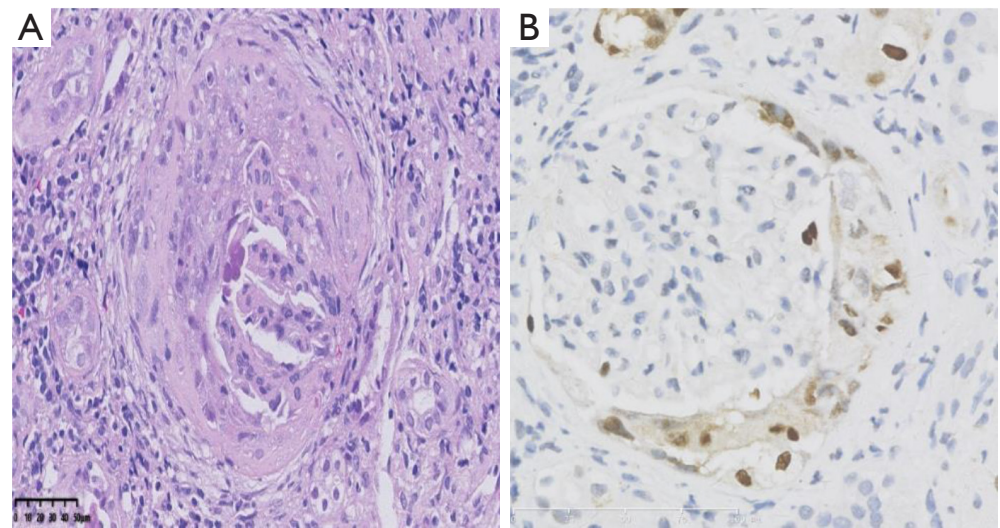

Figure 2 Crescent formation in BK polyomavirus-infected glomeruli. The proliferated glomerular parietal epithelial cells exhibited enlarged and ground-glass like nuclei (A, hematoxylin-eosin, $\times 400)$ and SV40 large T antigen expression (B, immunohistochemistry, $\times 400)$. 

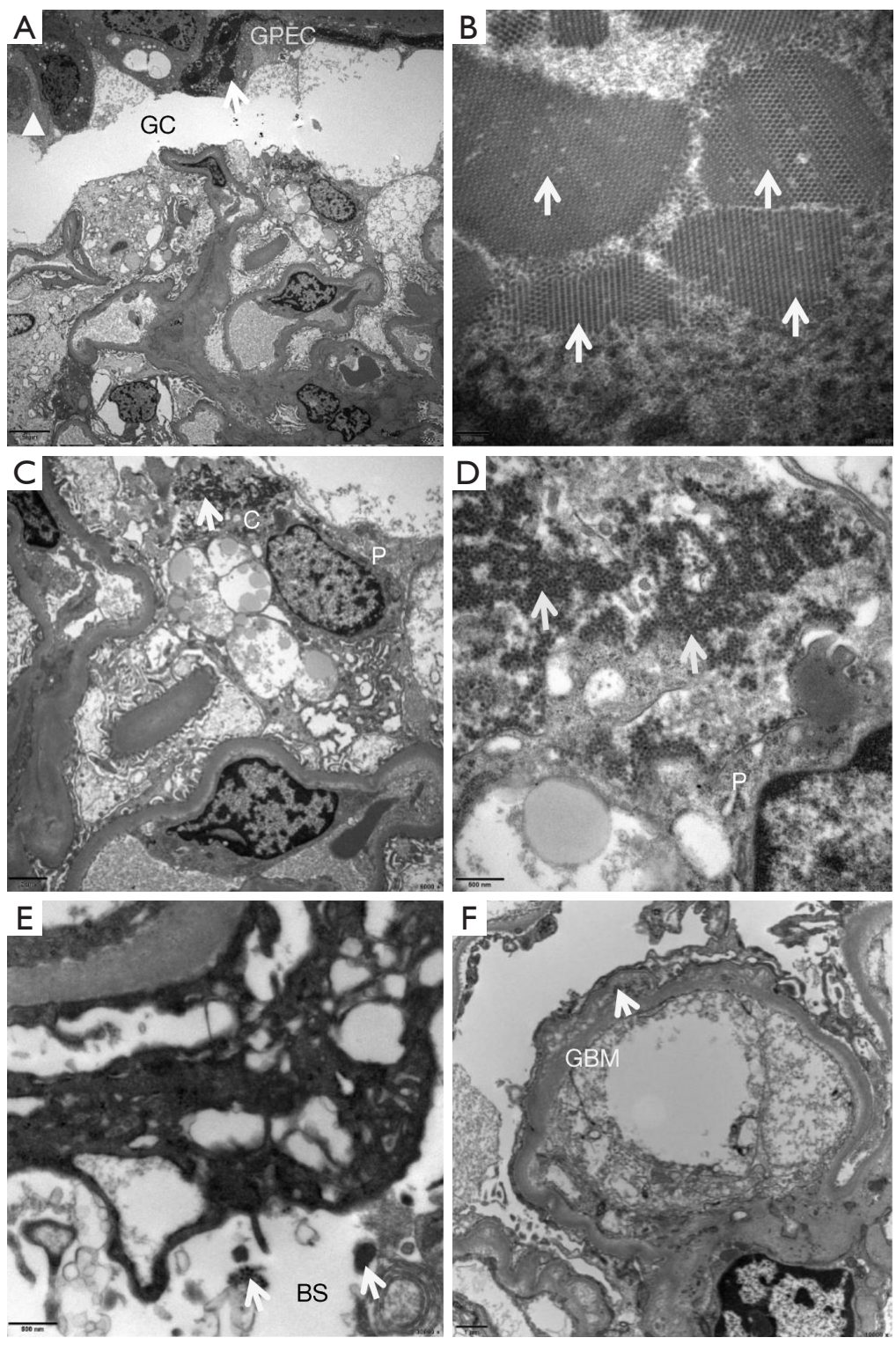

Figure 3 Ultrastructural changes of BK polyomavirus (BKPyV)-infected glomeruli. The involved glomerular capsule (GC) was thickened and layered (triangle), within which uniform dense structures or membranous cell debris (arrow) was found [A, electron microscopy (EM), $\times 2,500]$. The nuclei of glomerular parietal epithelial cells (GPEC) were enlarged and fusiform, with a large number of virus particles (arrows) (B, EM, $\times 40,000)$. Podocytes $(\mathrm{P})$ were occasionally infected by BKPyV. The uniform viral particles (arrows) were found inside the cytoplasm (C, EM, $\times 6,000)$, the foot processes (FP) (D, EM, ×20,000), Bowman's space (BS) (E, EM, $\times 20,000)$, and the glomerular basement membrane (GMB) (F, EM, ×10,000).

\section{Ultrastructural changes}

Each ultrathin section contained at least 1 non-sclerotic, non-ischemic glomerulus. Five (11.4\%) biopsy specimens had viral particles within the glomeruli. The capsular walls of the $\mathrm{BKPyV}$-infected glomeruli showed changes similar to those of the basement membranes of $\mathrm{BKPyV}$-infected tubules. The wall of the glomerular capsule was thickened and layered, with high electron-dense interposition, composed of uniform dense structures (Figure $3 A$ ) or membranous cell debris. Most $\mathrm{BKPyV}$-infected parietal epithelial cells were degenerative, with mitochondrial swelling, endoplasmic reticulum expansion, and multi- 
layered membranous structure formation. The nuclei were enlarged and fusiform with intact nuclear membranes. Large numbers of virus particles were found in the nucleus (Figure $3 B$ ) and cytomembrane of the partial epithelial cells, podocytes, and tubular epithelial cells. The viral particles were identified as tightly packed hexagonal arrays of naked, round, electron-dense structures that measure

Table 3 Results of immunofluorescence staining from 44 patients with glomerular BKPyV involvement

\begin{tabular}{lc}
\hline IF staining findings & $\mathrm{N}(\%)$ \\
\hline Glomerular mesangial area and capillary loops & $3(6.8)$ \\
IgA & $11(25.0)$ \\
IgM & $2(4.5)$ \\
IgG & $2(4.5)$ \\
C3 & $6(13.6)$ \\
C1q & $3(6.8)$ \\
Fibrinogen & \\
C4d deposition & $6(13.6)$ \\
Along GBM & $18(40.9)$ \\
Along TBM & 0 \\
Along ptc & \\
\hline BKPyV, BK polyomavirus; IF, immunofluorescence; GBM, \\
glomerular basement membranes; TBM, tubular basement \\
membranes; ptc, peri-tubular capillaries.
\end{tabular}

between 30 and $45 \mathrm{~nm}$ in diameter. The $\mathrm{BKPyV}$-infected visceral epithelial cells (podocytes) showed scattered virus particles in the cytoplasm (Figure 3C), and on the surface of the foot processes (Figure 3D). Typical viral particles were occasionally identified in Bowman's space (Figure $3 E$ ) and along the GBM (Figure 3F). No viral particles were found in the mesangial area, glomerular capillary lumen, or endothelial cells. There was no GBM breakage, foot process fusion, or electron-dense deposition in the mesangial or subendothelial areas.

\section{Repeat biopsies}

Twelve (27.3\%) patients received repeat biopsies within 1.6 to 39.5 months (median: 13.5 months) after the diagnosis of BKPyVAN. None of the repeat biopsies revealed persistent glomerular $\mathrm{BKPyV}$ involvement. SV40 large T antigen staining in the tubular epithelial cells became negative in 4 patients, and the degree of staining decreased remarkably in the other 8 patients. Two patients with cellular crescents received repeat biopsies, and the cellular crescents were found to be replaced by fibrous crescents, suggestive of late changes of previous epithelial proliferation. Compared with the initial biopsies, the repeat biopsies revealed decreased tubulitis and had lower interstitial inflammation scores, but exhibited increased tubular atrophy and interstitial fibrosis scores (Table 4). No repeat biopsy showed pathological signs of rejection.

Table 4 Comparison of pathological damage between initial biopsies and repeat biopsies

\begin{tabular}{|c|c|c|c|}
\hline Pathological lesions & Initial biopsy (N=12) & Repeat biopsy ( $\mathrm{N}=12)$ & $\mathrm{P}$ \\
\hline Glomerulitis & $0.5 \pm 0.3$ & $0.4 \pm 0.1$ & 0.572 \\
\hline Chronic allograft injury & 0 & $0.2 \pm 0.1$ & 0.328 \\
\hline Peri-glomerular fibrosis & $6 / 12$ & $8 / 12$ & 0.408 \\
\hline Fibrous crescents & $0 / 12$ & $2 / 12$ & 0.460 \\
\hline Extent of SV40 large T antigen & $2.5 \pm 0.9$ & $1.1 \pm 0.9$ & 0.001 \\
\hline Tubulitis & $1.3 \pm 0.5$ & $1.1 \pm 0.9$ & 0.408 \\
\hline Interstitial inflammation & $1.3 \pm 1.2$ & $0.6 \pm 0.9$ & 0.103 \\
\hline
\end{tabular}




\section{Discussion}

In a review of 124 biopsies, Celik et al. reported that 29\% of biopsies had glomerular BKPyV infection (4). Similarly, in our study, glomerular $\mathrm{BKPyV}$ infection was identified in $24.4 \%(44 / 180)$ of the biopsy specimens, suggesting that glomerular $\mathrm{BKPyV}$ involvement is not uncommon. In general, medullary collecting ducts and distal tubules are involved at the onset of BKPyVAN (7). Occasionally, virus-associated cytopathic features can also be noted in the GPECs, especially in severely infected cases and at later stages (12). This pathological phenomenon may suggest a worse prognosis.

Crescentic glomerulonephritis is uncommon in renal transplant recipients. Most cases are caused by recurrent disease, and less frequently, by de novo disease (13). Preexisting crescents from the donor kidney also can be identified in the transplanted kidney in early posttransplantation biopsy specimens (14). Rarely, pathogen infection-related crescents have been reported in cases of streptococcus (15), cytomegalovirus (16), and Epstein-Barr virus infection (17). In several case reports (18), the presence of glomerular crescents harboring $\mathrm{BKPyV}$-infected cells has been described in renal transplant recipients. It is known that crescents are formed after breakage of capillary loops and fibrin leakage into Bowman's space, leading to parietal epithelial cell proliferation. In the present study, there was no evidence of capillary loop breakage or fibrin leakage into Bowman's space. In addition, immune deposits were absent in glomeruli under both IF microscopy and electron microscopy. We had assumed that crescent formation might be due to $\mathrm{BKPyV}$ infection or recurrence of the primary kidney disease. Laboratory examination and repeat biopsies ruled out the latter possibility. Therefore, it is most likely that crescents represent a florid proliferation of GPECs induced by direct infection of $\mathrm{BKPyV}$ through contiguous spread from the adjacent proximal tubule. Positive SV40 large $\mathrm{T}$ antigen staining within glomerular crescents and adjacent proximal tubules support a hypothesis that $\mathrm{BKPyV}$-associated tubule-interstitial nephritis and crescent formation are causally related (13). Therefore, we suggest that $\mathrm{BKPyV}$ infection should be included in the differential diagnosis of crescentic glomerulonephritis in KT recipients to avoid unnecessary modulation of immunosuppressants.

The fact that 19 biopsy specimens with Banff $\mathrm{g} 1$ glomerulitis, of which 11 had concurrent peri-tubular capillaritis calls for comment. The coexistence of glomerulitis and peri-tubular capillaritis may suggest antibody-mediated rejection (ABMR) (10). However, since C4d deposition along the peri-tubular capillaries was absent, and donorspecific antibody was negative, a diagnosis of ABMR is unlikely. Glomerulitis has been reported in other viral infections, such as cytomegalovirus (16), hepatitis virus (19), and B19 virus (20). In our long-term observations, we found that some BKPyVAN cases, with or without glomerular involvement, had mild lymphocyte infiltration in glomerular and peri-tubular capillaries. Mild glomerulitis in BKPyVinfected glomeruli in this study might be associated with viral replication in the glomeruli, and the immune response against $\mathrm{BKPyV}$. However, this supposition requires further investigation.

The identification of intra-nuclear inclusions by light microscopy and positive IHC staining for SV40 large T antigen establishes a diagnosis of BKPyVAN. Electron microscopy can severe as a supplement to diagnosis via direct visualization of viral particles within tubule epithelium and glomeruli. Celik et al. examined the glomerular ultrastructure in 16 biopsy specimens from renal transplant recipients with BKPyVAN, but no viral particles were detected (4). In our study, viral particles within the glomeruli were found in only $11.4 \%$ of all BKPyVAN biopsy specimens. These data indicate that finding evidence of $\mathrm{BKPyV}$ in glomeruli by electron microscopy is challenging. Interestingly, in one biopsy specimen, we found viral particles in GPECs and podocytes. The process of BKPyV infection in parietal epithelial cells and podocytes is similar to the process of $\mathrm{BKPyV}$ infection in tubular cells described in detail by Drachenberg et al. (21). In a case report, BKPyV was identified within the GBM presenting as subepithelial depositions, suggesting a mechanism for antigen clearance via deposition formation and cytoplasmic processing by podocytes (22). This phenomenon was also observed in our study. In one of our cases, electron microscopy revealed that virus particles were enveloped by protein casts within the glomerular space. However, no virus particles were found in the mesangial area, glomerular capillary lumen, or endothelial cells. Therefore, we can speculate that virus particles shed from BKPyV-infected tubular epithelial cells formed a complex with Tamm-Horsfall protein (THP) in the tubule, which was occasionally refluxed into the glomerular space (23), eventually resulting in glomerular involvement. This finding partially supports the speculation that the distribution of $\mathrm{BKPyV}$ within the glomerulus is mainly derived from the adjacent tubular epithelium.

It is believed that $\mathrm{BKPyV}$ is initially latent in the ureteral transitional epithelium (24). Under powerful 
immunosuppression, the virus activates and spreads in an ascending manner via from collecting ducts to distal tubules and proximal tubules (7). If the infection is not effectively controlled, the virus will eventually infect the glomeruli, since the glomerular capsule is contiguous with the proximal tubules. Bedside, $\mathrm{BKPyV}$ can easily infect Bowman's capsular epithelium because it has the same origin as tubular epithelium, i.e., the nephrogenic vesicle.

In conclusion, $\mathrm{BKPyV}$ can infect the glomeruli and causes a series of glomerular pathological changes. However, the correlation between this unique pathological phenomenon and clinical prognosis needs further study.

\section{Acknowledgments}

The authors are grateful to the nursing staff of our center for collaboration and dedication to the patients.

Funding: This work was supported by grants from the National Natural Science Foundation of China (81770749); the Natural Science Foundation of Guangdong Province (2017A030313710); the China Organ Transplantation Development Foundation (2019JYJH07); and the Fundamental Research Funds for the Central Universities (17ykpy29). We would like to give special thanks to the Guangdong Provincial Key Laboratory of Organ Donation and Transplant Immunology (2013A061401007, 2017B030314018), and the Guangdong Provincial International Cooperation Base of Science and Technology (Organ Transplantation) (2015B050501002) for providing experimental platforms.

\section{Footnote}

Reporting Checklist: The authors have completed the STROBE reporting checklist. Available at http://dx.doi. org/10.21037/atm-20-1879

Data Sharing Statement: Available at http://dx.doi. org/10.21037/atm-20-1879

Conflicts of Interest: All authors have completed the ICMJE uniform disclosure form (available at http://dx.doi. org/10.21037/atm-20-1879). The authors have no conflicts of interest to declare.

Ethical Statement: The authors are accountable for all aspects of the work in ensuring that questions related to the accuracy or integrity of any part of the work are appropriately investigated and resolved. The study was conducted in accordance with the Declaration of Helsinki (as revised in 2013). The study was approved by the Ethics Committee and the Research Board of our institution [Approval No. (2009) 28]. Because of the retrospective nature, the requirement of informed consent was waived.

Open Access Statement: This is an Open Access article distributed in accordance with the Creative Commons Attribution-NonCommercial-NoDerivs 4.0 International License (CC BY-NC-ND 4.0), which permits the noncommercial replication and distribution of the article with the strict proviso that no changes or edits are made and the original work is properly cited (including links to both the formal publication through the relevant DOI and the license). See: https://creativecommons.org/licenses/by-nc-nd/4.0/.

\section{References}

1. Drachenberg CB, Papadimitriou JC, Chaudhry MR, et al. Histological Evolution of BK Virus-Associated Nephropathy: Importance of Integrating Clinical and Pathological Findings. Am J Transplant 2017;17:2078-91.

2. Nickeleit V, Singh HK, Randhawa P, et al. The Banff Working Group Classification of Definitive Polyomavirus Nephropathy: Morphologic Definitions and Clinical Correlations. J Am Soc Nephrol 2018;29:680-93.

3. Lusco MA, Fogo AB, Najafian B, et al. AJKD Atlas of Renal Pathology: Polyomavirus Nephropathy. Am J Kidney Dis 2016;68:e37-8.

4. Celik B, Randhawa PS. Glomerular changes in BK virus nephropathy. Hum Pathol 2004:35:367-70.

5. Hariharan S. BK virus nephritis after renal transplantation. Kidney Int 2006;69:655-62.

6. Chen XT, Yang SC, Chen WF, et al. Glomerular Parietal Epithelial Cells Infection Is Associated With Poor Graft Outcome in Kidney Transplant Recipients With BK Polyomavirus-Associated Nephropathy. J Infect Dis 2019;219:1879-86.

7. Hirsch HH, Randhawa P; AST Infectious Diseases Community of Practice. BK polyomavirus in solid organ transplantation. Am J Transplant 2013;13 Suppl 4:179-88.

8. Huang G, Wu LW, Yang SC, et al. Factors Influencing Graft Outcomes Following Diagnosis of Polyomavirus -Associated Nephropathy after Renal Transplantation. PLoS One 2015;10:e0142460.

9. Huang G, Wang CX, Zhang L, et al. Monitoring of polyomavirus $\mathrm{BK}$ replication and impact of preemptive 
immunosuppression reduction in renal-transplant recipients in China: a 5-year single-center analysis. Diagn Microbiol Infect Dis 2015;81:21-6.

10. Haas M, Loupy A, Lefaucheur C, et al. The Banff 2017 Kidney Meeting Report: Revised diagnostic criteria for chronic active $T$ cell-mediated rejection, antibodymediated rejection, and prospects for integrative endpoints for next-generation clinical trials. Am J Transplant 2018;18:293-307.

11. Sis B, Mengel M, Haas M, et al. Banff '09 meeting report: antibody mediated graft deterioration and implementation of Banff working groups. Am J Transplant 2010;10:464-71.

12. Drachenberg CB, Hirsch HH, Ramos E, et al.

Polyomavirus disease in renal transplantation: review of pathological findings and diagnostic methods. Hum Pathol 2005;36:1245-55.

13. Nair R, Katz DA, Thomas CP. Diffuse glomerular crescents and peritubular immune deposits in a transplant kidney. Am J Kidney Dis 2006;48:174-8.

14. Kiser RL, Thomas DB, Andreoni K, et al. Preexisting crescentic glomerulonephritis in the renal allograft. Am J Kidney Dis 2003;42:E20-6.

15. Hikita T, Arai K, Inokami T, et al. A case of fulminant acute poststreptococcal glomerulonephritis showing mesangiolysis and crescent formation preceded by erysipelas. Nihon Jinzo Gakkai Shi 2002;44:558-63.

16. Detwiler RK, Singh HK, Bolin P Jr, et al. Cytomegalovirus-induced necrotizing and crescentic glomerulonephritis in a renal transplant patient. Am J

Cite this article as: Chen XT, Deng RH, Yang SC, Hou XT, Li J, Chen YY, Yang HF, Wang ZY, Wang CX, Qiu J, Chen LZ, Chen WF, Huang G. Pathological characteristics of BK polyomavirus-associated nephropathy with glomerular involvement. Ann Transl Med 2020;8(15):923. doi:10.21037/ atm-20-1879
Kidney Dis 1998;32:820-4.

17. Mirsaeidi M, Syed F, Jaffe ES. Antineutrophil Cytoplasmic Autoantibody Associated Systemic Vasculitis Is Associated with Epstein - Barr virus in the Setting of HIV Infection. Infect Dis Clin Pract (Baltim Md) 2013;21:50-3.

18. Yoon HJ, Sun IO, Yeo MK, et al. Severe Crescentic BK Nephropathy: A Case Report. J Korean Soc Transplant 2016;30:35-7.

19. Yamabe H, Nakamura N, Nakamura $M$, et al. Hepatitis $C$ virus-associated glomerulonephritis without hepatitis $\mathrm{C}$ virus in the blood. Am J Kidney Dis 2005;46:e65-9.

20. Nakazawa T, Tomosugi N, Sakamoto K, et al. Acute glomerulonephritis after human parvovirus B19 infection. Am J Kidney Dis 2000;35:E31.

21. Drachenberg CB, Papadimitriou JC, Wali R, et al. BK polyoma virus allograft nephropathy: ultrastructural features from viral cell entry to lysis. Am J Transplant 2003;3:1383-92.

22. Brealey JK. Ultrastructural observations in a case of BK virus nephropathy with viruses in glomerular subepithelial humps. Ultrastruct Pathol 2007;31:1-7.

23. Kawanishi K, Honda K, Koike J, et al. A Preliminary Study Into the Significance of Intrarenal Reflux in BK Virus Nephropathy After Kidney Transplantation. Transplant Direct 2016;2:e64.

24. Lamarche C, Orio J, Collette S, et al. BK Polyomavirus and the Transplanted Kidney: Immunopathology and Therapeutic Approaches. Transplantation 2016;100:2276-87. 



Figure S1 Glomerulus involved by BK polyomavirus presenting with glomerulitis. In this glomerulus, a cellular crescent was formed, composed of the proliferated parietal epithelial cells with nuclear enlargement and intra-nucleic inclusion. Meanwhile, segmental glomerulitis can be identified with mononuclear cell infiltrates and endothelium swelling (arrow) (hematoxylin-eosin, $\times 400$ ). Immunohistochemistry staining revealed SV40 large T antigen expression in the proliferated parietal epithelium (immunohistochemistry, $\times 400)$.

Table S1 Urine test results in 44 patients with glomerular BKPyV involvement

\begin{tabular}{lccc}
\hline Urine-related indicators & Median & Upper quartile & Lower quartile \\
\hline Urine volume $(\mathrm{mL} / 24 \mathrm{~h})$ & 2,700 & 2,000 & 3,000 \\
Urine specific gravity & 1.008 & 1.006 & 1.009 \\
Urinary red blood cell $(/ \mathrm{hL})$ & 3.0 & 1.0 & 7.9 \\
Urine protein quantitation $(\mathrm{g} / 24 \mathrm{~h})$ & 0.316 & 0.227 & 0.500 \\
Urine microalbumin $(\mathrm{mg} / \mathrm{L})$ & 24.10 & 9.45 & 68.78 \\
Urine albumin-to-creatinine ratio & 53.0 & 25.5 & 134.5 \\
\hline
\end{tabular}

BKPyV, BK polyomavirus. 